\title{
6 COMUNICACIÓN ORGANIZACIONAL ANTE EL RETO DE LA ESTRATEGIA MULTIPLATAFORMA Y 2.0: LA EXPERIENCIA DE LOS PARTIDOS POLÍTICOS EN EL PAÍS VASCO
}

\author{
Organisational communication facing the challenge of \\ multiplatform and Web 2.0 strategy: The experience \\ of the political parties in the Basque Country
}

Ainara Larrondo-Ureta

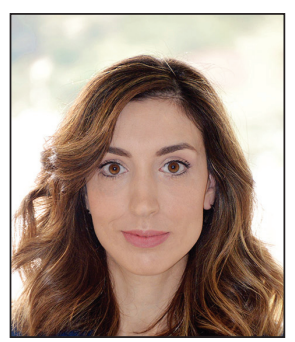

Ainara Larrondo-Ureta, doctora en periodismo y máster en historia contemporánea, es profesora y vicedecana en la Facultad de Ciencias Sociales y de la Comunicación de la Universidad del País Vasco-Euskal Herriko Unibertsitatea (UPV/EHU). Ha sido investigadora visitante en el Centre for Cultural Policy Research (CCPR) de la University of Glasgow (2012) y ha publicado numerosos trabajos en torno al impacto de la comunicación digital en la práctica periodística, desde el punto de vista del mensaje y del trabajo en las redacciones. Como miembro de una decena de proyectos de investigación competitivos (Ministerio, Gobierno Vasco, UPV/EHU), ha analizado también problemáticas vinculadas al fenómeno de la convergencia multimedia, las audiencias activas, el periodismo móvil o la comunicación organizacional online.

http://orcid.org/0000-0003-3303-4330

Universidad del País Vasco (UPV/EHU), Facultad de Comunicación, Despacho Decanato 2.8 Barrio Sarriena, s/n. 48940 Campus de Leioa (Vizcaya), España ainara.larrondo@ehu.es

\section{Resumen}

Conscientes de que en la sociedad actual transmitir información resulta insuficiente para comunicar con éxito, las organizaciones tienden a ser cada vez más emisores múltiples, además de receptores de lo que expresan sus públicos. De manera particular, las organizaciones políticas y sus líderes han venido mostrando en los últimos años gran interés por explorar usos comunicativos tecnológicamente avanzados, en sintonía con la idea de campaña permanente (Blumenthal, 1980; Ornstein; Mann, 2000). En este escenario, el artículo se pregunta por las tendencias que marcan actualmente la gestión de la comunicación política fuera de contextos puntuales electorales, tomando como caso de estudio cinco partidos con representación parlamentaria en la Comunidad Autónoma Vasca (CAV). A partir de una metodología cualitativa sustentada en el análisis de sus websites organizacionales y en entrevistas en profundidad con sus Departamentos de Comunicación, el artículo explica por qué estas organizaciones políticas se encuentran en un escenario evolutivo 1.5 (Kalnes, 2009).

\section{Palabras clave}

Comunicación externa; Organizaciones; Partidos políticos; Sitios web; Web 2.0; País Vasco; España.

\begin{abstract}
Aware of the fact that nowadays transmitting information is insufficient for successfully communicating, organizations are increasingly multi-channeled communicators, in addition to receiving what their public communicates, because they are aware that simply transmitting information is no longer sufficient for successful communication. In particular, political organizations and their leaders have shown a strong interest in recent years for exploring technologically advanced communication avenues, in accordance with the 'permanent campaign' idea (Blumenthal, 1980; Ornstein; Mann, 2000). This article examines current tendencies that determine the management of political communication, outside of specific voting contexts, through a case study of five parties with parliamentary representation in the Autonomous Community of the Basque Country, Spain. Using qualitative methodology, based on website analysis and in-depth interviews with press departments, this study explains why these organizations situate themselves in a 1.5 evolutionary phase (Kalnes, 2009).
\end{abstract}

\section{Keywords}

External communication; Organizations; Political parties; Websites; Web 2.0; Basque Country; Spain. 
Larrondo-Ureta, Ainara (2016). “Comunicación organizacional ante el reto de la estrategia multiplataforma y 2.0: la experiencia de los partidos políticos en el País Vasco". El profesional de la información, v. 25, n. 1, pp. 114-123.

http://dx.doi.org/10.3145/epi.2016.ene.11

\section{Introducción}

El escenario comunicativo actual está marcado por múltiples retos y oportunidades, también en lo que se refiere a la comunicación organizacional externa. Como en otros ámbitos, en este los procesos más tradicionales mantienen su peso, al tiempo que se realizan constantes esfuerzos por mejorar los usos de las herramientas web para alcanzar un verdadero escenario 2.0 .

Este análisis de los mecanismos actuales de la comunicación externa ha encontrado en la política un ámbito de particular interés (Canel; Zamora, 2004). No en vano los partidos se enfrentan desde hace años a una crisis de imagen, representatividad y confianza que no ha hecho sino aumentar la exigencia de su comunicación con la ciudadanía. Con todo, la comunicación de los partidos políticos parece estar lejos del grado de desarrollo alcanzado en las grandes corporaciones; además de existir déficits en las relaciones con los medios de comunicación de tipo vertical, se desaprovechan oportunidades en los usos online para la comunicación horizontal y la interacción en directo, ya se trate de sitios web organizacionales o de plataformas 2.0 (Gómez-Castellanos; Ortiz-Marín; Concepción-Montiel, 2011, p. 73).

Los partidos se enfrentan desde hace años a una crisis de imagen, representatividad y confianza que no ha hecho sino aumentar la exigencia de su comunicación con los ciudadanos

A diferencia del ámbito offline, donde la comunicación opera de manera jerárquica, desde las organizaciones políticas hacia los medios y desde éstos hacia los ciudadanos (esquema oneto-many), en el ámbito online el público funciona al mismo tiempo como emisor y receptor (emirec), o como productor y consumidor (prosumidor). Ello obliga a los emisores tradicionales -departamentos de comunicación y medios de masas- a compartir la propiedad de la información y a convertirse, a su vez, en receptores de lo que expresan los públicos y las audiencias activas (esquema many-to-many). Relacionado con ello, cabría recordar también que el uso combinado de diferentes medios ha contribuido a diversificar la disímil audiencia de la comunicación política, conformada por los ciudadanos en general, por grupos afines con un especial sentido de pertenencia, y por los propios medios de comunicación y los periodistas, a quienes se suman hoy otro tipo de comunicadores influencers.

Este paradigma suaviza así la tradicional dependencia hacia los medios de comunicación, responsable en cierta medida del distanciamiento entre los partidos políticos y sus públi$\cos 0$, si se prefiere, de la apatía y rechazo generalizado de los ciudadanos hacia la política. Se ha entendido que los medios de masas favorecen la pasividad y el alejamiento ante los problemas (disfunción narcotizadora), además de mostrar una excesiva aquiescencia hacia el departamento de comunicación en la relación periodistas-políticos. Asimismo, los medios tienden a personalizar el mensaje político en la figura del líder, dejando de lado en muchas ocasiones los ideales y las propuestas.

Profundizar en estos usos tecnopolíticos y evaluar hasta qué punto se está respondiendo en el día a día a dicha especificidad representa así una prioridad, tanto a nivel profesional, como académico (Gutiérrez-Rubí, 2014). Los departamentos de comunicación de los partidos asumen hoy la necesidad de orquestar múltiples acciones y de hacer converger éstas en la búsqueda de un mismo resultado. De hecho, se percibe desde hace dos décadas en estos departamentos una clara evolución marcada por estrategias específicas para el medio con mayor poder de proyección, la Web (García-Orosa, 2009; Losada-Díaz, 2009; Túñez-López, 2012).

La última etapa se iniciaba hace ahora aproximadamente una década. En ésta, aunque los sitios web continúan funcionando como una herramienta clave en la imagen y la comunicación organizacional, el departamento se suma a la agitación generada por la expansión de la web 2.0 y se posiciona en las plataformas con mayor empuje social (Twitter, Facebook, Blogger, Instagram, etc.). Se percibe así que los partidos comienzan a tomarse en serio la campaña online, más allá de los sitios web.

Esta evolución a nivel de gestión comunicativa externa se ha vinculado de manera particular al fenómeno de la distribución multiplataforma y 2.0. Ello obliga a prestar atención al trabajo de los departamentos implicados en la producción y gestión del mensaje político, una línea de investigación que ha resultado hasta la fecha escasamente explorada, a pesar del valor de la actividad comunicativa regular para la política.

\section{Antecedentes}

La investigación en comunicación política ha demostrado ser un campo de estudio fecundo y dinámico, capaz de prestar interés a perspectivas clásicas desde planteamientos renovados. Éstos se han centrado en el análisis del contenido político que ofrecen los medios online y en el impacto de dicho contenido sobre las audiencias activas. Asimismo, se ha dedicado un buen número de estudios al análisis de la conocida como ciberpolítica, especialmente a través de las e-campañas, cibercampañas o campañas hipermedia (Howard, 2006). Por razones de espacio, no nos extenderemos en esta cuestión, si bien han sido numerosos los autores que han abordado este fenómeno (Schweitzer, 2008; Vaccari, 2008; Dader et al., 2014; etc.).

Estas investigaciones ponen de manifiesto la utilidad de los sitios web organizacionales como herramienta empírica 
para profundizar en la comunicación regular de los partidos (Padró-Solanet; Cardenal, 2008, p. 48; De-Landtsheer, 2012). De hecho, la actividad de los sitios web políticos varía sustancialmente dentro y fuera de los períodos electorales (Vaccari, 2008; Gibson et al., 2003; ValeraOrdaz; López-García, 2014), y lo mismo podría decirse del uso de las redes sociales (Vergeer; Hermans; Sams, 2011; Spyridou; Veglis, 2011; Macnamara; Sakinofsky; Beattie, 2012).

En general, todos los estudios referidos ponen de manifiesto que la adaptación de los partidos a una nueva era de la comunicación digital ha resultado en muchos casos más difícil de lo que se podía pensar en un principio. Entre otras causas, las webs organizacionales políticas han presentado escasos espacios para el ejercicio de la transparencia, la interactividad y la discusión ciudadana, en contraste con los materiales de refuerzo, mucho más abundantes (ideario, historia, programa, etc.). Más recientemente, el análisis de Catarina Silva (2013) en torno a las webs de los partidos portugueses demuestra usos interactivos todavía limitados, en comparación con las expectativas generadas en torno a la capacidad de diálogo del medio online.

\section{Metodología}

En función del estado del arte descrito, se decidió centrar la investigación en la actividad de comunicación externa política fuera de campaña y en un contexto político-social de carácter regional, siguiendo el ejemplo de estudios anteriores, como el elaborado por Padró-Solanet y Cardenal (2008) en torno a los partidos catalanes. Con este fin se optó por el estudio de caso, dada la utilidad de esta técnica para analizar en profundidad el fenómeno y establecer tendencias aplicables a otros casos de características similares.

El estudio se centró concretamente en las cinco organizaciones políticas representadas en el Parlamento Vasco en la legislatura 2012-2016:

- Euskal Alberdi Jeltzaleak-Partido Nacionalista Vasco (EAJPNV)

- Euskal Herria Bildu (EH Bildu)

- Partido Socialista de Euskadi-Euskadiko Ezkerra (PSE-EE/ PSOE)

- Partido Popular Vasco-Euskal Talde Popularra (PP País Vasco)

- Unión Progreso y Democracia (UPyD País Vasco)
Esta muestra se consideró adecuada por cubrir espectros políticos diferenciados y partidos de diferentes tamaños, distinguidos tanto por su tradición, como por su novedad. En la elección de este caso de estudio se tuvo en cuenta que la comunicación política regular resulta especialmente importante en aquellas sociedades para las que las preocupaciones o problemas de tipo político ocupan un lugar preferente (Canel; Zamora, 2004), como ocurre en el País Vasco (Gobierno Vasco, 2012).

\section{Es necesario experimentar más con cam- pañas de tipo bottom-up en momentos electorales puntuales}

De manera concreta, la investigación ha buscado describir el plan estratégico que guía el trabajo de los departamentos o gabinetes de comunicación de estos partidos al tener en cuenta, según la hipótesis de partida, que su estrategia vive un momento de cambio en el que el uso de las herramientas tradicionales para transmitir a los medios-periodistas y los ciudadanos sigue manteniendo un protagonismo importante, aunque compartido cada vez más con el sitio web organizacional y las plataformas 2.0.

En función de esta premisa y del objetivo principal del estudio, se emplearon diversas técnicas de análisis. Entre los 
meses de diciembre de 2014, enero, febrero y marzo de 2015 se llevó a cabo una evaluación de los recursos web y las funciones de sus sitios organizacionales, en tanto que eje central de su estrategia comunicativa online:

\section{- EAJ-PNV http://www.eaj-pnv.eus/esp \\ - EH BILDU http://ehbildu.eus/es \\ - PP País Vasco http://ppvasco.com \\ - PSE-EE / PSOE \\ http://www.socialistasvascos.com \\ - UPyD País Vasco http://www.upyd.es/Pais-Vasco}

Mientras la comunicación en los medios sociales resulta todavía hoy bastante dependiente de las comunicaciones y mensajes distribuidos mediante otros canales, el sitio web es considerado un espacio de comunicación autónomo y con gran potencial.

Concretamente se tuvieron en cuenta las cuatro dimensiones o funciones principales de los sitios web organizacionales políticos (comunicación-movilización-interacciónaccesibilidad), siguiendo diversas propuestas metodológicas sustentadas en la funcionalidad de este tipo de sitios (Gibson; Ward, 2000; Gibson et al., 2003; Padró-Solanet; Cardenal, 2008, p. 55; De-Landtsheer, 2012; Silva, 2013, pp. 204-207).

Para analizar en qué medida se cumplen o no dichas funciones, se recurrió a una ficha de codificación con cuarenta y nueve indicadores de naturaleza dicotómica (1=Sí existe; $2=$ No existe). Dichos indicadores, observables desde el punto de vista del usuario, aparecen especificados en las tablas que acompañan a este artículo. Desde un punto de vista más general, estos indicadores tienen también relación con aspectos básicos en torno a las webs, como son:

- diseño (usabilidad y accesibilidad);

- forma del contenido (hipertextualidad y multimedialidad);

- calidad (actualización, servicios tipo buscador, hemeroteca, etc.);

- interactividad.

En relación con este último indicador se tuvieron en cuenta las opciones que permiten al usuario participar del contenido de distintas maneras: envío de materiales, aportación de comentarios y acceso a plataformas sociales para la dinamización del contenido (Twitter, Facebook, RSS, Google +, Instagram, Flickr, LinkedIn, etc.).
Por su claridad y demostrada eficacia, se ha considerado también la metodología de análisis y evaluación de los recursos online propuesta por Lluís Codina (2006), centrada en parámetros que condicionan el consumo del contenido por parte del público, como la accesibilidad y navegabilidad de los sitos web.

Al examen descrito se sumaron ocho entrevistas semiestructuradas, realizadas en el primer semestre de 2015. Estas conversaciones buscaron completar los datos del análisis de las webs a partir de las visiones de quienes se encargan diariamente de la planificación y gestión de su contenido, esto es, los responsables de comunicación o dircoms, y los profesionales relacionados directamente con el trabajo de comunicación online. Las preguntas giraron en torno a la visión y los objetivos del departamento, así como a su estrategia y gestión de los principales espacios online en los que está hoy presente el partido: el sitio web organizacional y las plataformas de medios 2.0.

Si bien el análisis de los sitios web organizacionales no contempló el examen específico de los contenidos aportados por los partidos y los usuarios en los principales espacios interactivos, sí se consideró oportuno emplear las entrevistas para profundizar, además de en los fines generales del sitio web, en aquellas cuestiones relativas a la presencia concreta en los medios sociales (transmitir una imagen po- 
sitiva, mejorar la reputación digital, etc.). Este interés responde al modelo de comunicación corporativa hoy en boga, definido como interactivo y social, y a su particular utilidad para los fines comunicativos de organizaciones como los partidos políticos.

\section{Análisis de resultados}

\subsection{Adaptación organizativa y profesional}

Los departamentos de comunicación examinados cuentan con equipos de trabajo reforzados hacia el año 2008 a partir de la incorporación de nuevos profesionales con perfil técnico o multimedia. Aquellos departamentos que cuentan con equipos dedicados en exclusiva al ámbito de internet evidencian una especialización de funciones en tres áreas concretamente:

- gestión de la información web y/o edición audiovisual;

- diseño de la estrategia web y 2.0;

- gestión y monitoreo de medios sociales (community management).

Esta especialización no abarca otros ámbitos punteros como el posicionamiento SEO (search engine optimization). Ello no impide, sin embargo, que algunos partidos hayan comenzado a conceder relevancia a esta estrategia:

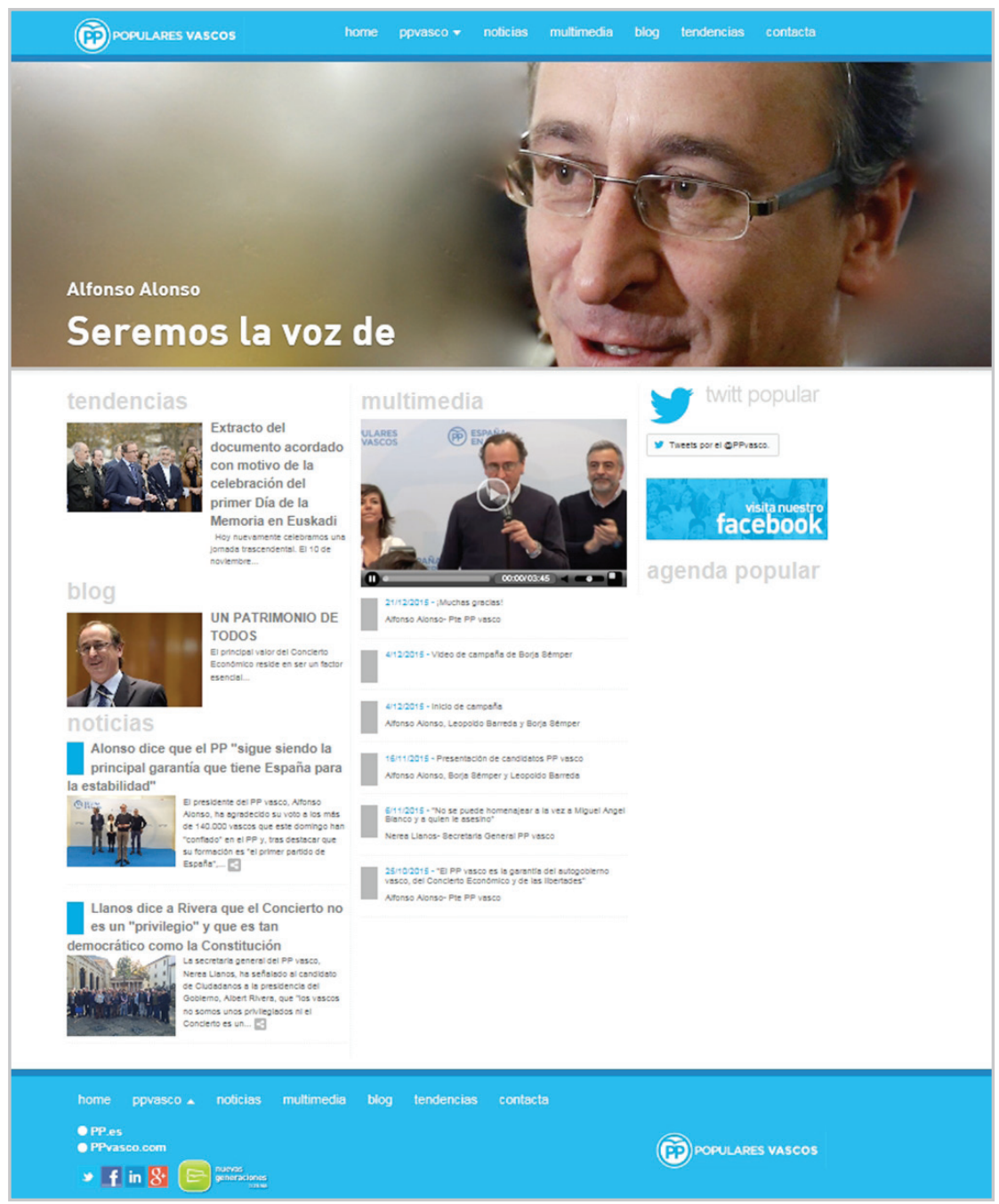

http://ppvasco.com
“Más allá del metatexto y el algo-

ritmo, el SEO es una estrategia indispensable para conseguir una reputación online y ser visible de la manera más positiva posible" (entrevista a EAJ-PNV).

Todos los departamentos analizados priorizan perfiles polivalentes, lo cual se convierte en un requisito para aquellos profesionales que trabajan en los departamentos más reducidos, caso de UPyD País Vasco o EH Bildu. Los dircoms entrevistados reconocen que han asumido una nueva mentalidad, polivalente y cada vez más proclive a los intereses de los públicos de la organización, poniendo en marcha acciones estratégicas acordes, si bien prefieren no autodefinirse como dircoms 2.0. De hecho, coinciden en admitir que no poseen un perfil especializado y que su nivel de competencia en el manejo de las herramientas sociales resulta todavía en algunos casos limitado, aunque en su trabajo tengan en cuenta el factor 2.0, siempre dentro de un conjunto de acciones más amplio.

\subsection{Visión del trabajo de comunicación}

Si bien no todos los partidos examinados cuentan con un plan de comunicación como tal, sí han desarrollado líneas concretas de actuación, acciones que dan forma a un plan de comunicación en la práctica diaria, más que sobre papel.

Los departamentos reconocen que sus intereses comunicativos están cada vez más focalizados en los espacios de la web y que este interés requiere aún de una mayor reflexión y concreción. Para ello, partidos como EAJ-PNV y PP Vasco han decidido apoyarse en servicios externos puntuales relacionados con el marketing y la publicidad, desde un punto de vista de estrategia y análisis. Todas las organizaciones consideradas otorgan gran importancia a la web, si bien son aquellas agrupaciones de más reciente creación -EH Bildu y UPyD País Vasco- las que confieren un reconocimiento especial al rol de este medio. Por su parte, las corporaciones con mayor recorrido han dejado de evidenciar una clara preferencia por los medios tradicionales y tienden a priorizar una visión multiplataforma, en la que todos los usos resultan estratégicos, aunque depositen un especial entusiasmo en las virtudes y posibilidades del medio web:

"No se trata de pasar sin ningún tipo de criterio a una especie de hiperactividad, pero está claro que es necesario explotar en mayor medida las oportunidades que tiene este ámbito" (entrevista a PP Vasco).

Según admiten estos departamentos, internet es un ámbito particular que ha introducido cambios de forma y fondo en sus fines y funcionamiento, más allá del uso de unas u otras herramientas:

"Nos dedicamos mucho al cómo emitimos en Internet, pero deberíamos dedicarnos más al cómo se recibe el mensaje, a saber cómo es la recepción y qué efectos tiene... si no llega o si genera rechazo, la utilidad con la que 
Tabla 1. Dimensiones de los sitios web. Resumen de resultados porcentuales por categoría de análisis y partido

\begin{tabular}{|c|c|c|c|c|c|}
\hline \multirow[b]{2}{*}{ Dimensiones } & \multicolumn{5}{|c|}{ Partido } \\
\hline & $\begin{array}{c}\text { EAJ-PNV } \\
\text { http://www.eaj-pnv. } \\
\text { eus/esp }\end{array}$ & $\begin{array}{c}\text { EH BILDU } \\
\text { http://ehbildu.eus/es }\end{array}$ & $\begin{array}{c}\text { PP vasco } \\
\text { http://ppvasco.com }\end{array}$ & $\begin{array}{c}\text { PSE-EE / PSOE } \\
\text { http://WWW.socialistasvas- } \\
\text { cos.com }\end{array}$ & $\begin{array}{c}\text { UPyD } \\
\text { http://www.upyd.es/Pais- } \\
\text { Vasco }\end{array}$ \\
\hline Comunicación & 32,35 & 11,74 & 29,41 & 17,64 & 8,82 \\
\hline Movilización & 23,07 & 10,25 & 20,51 & 22,58 & 25,64 \\
\hline Interacción & 26,00 & 16,00 & 18,00 & 20,00 & 20,00 \\
\hline Accesibilidad & 25,80 & 9,67 & 25,80 & 20,51 & 16,12 \\
\hline
\end{tabular}

quiere presentarse el partido ante la sociedad se pierde, porque lo que emite se entiende como no útil... somos lo que comunicamos" (entrevista a PSE-EE / PSOE)

El hecho de que hoy cualquier usuario pueda ser generador de información obliga a entender el mapa de relaciones y de control de la información de una manera diferente, de ahí que reconozcan que su relación tradicional con los medios del entorno ha cambiado:

'Los gabinetes de comunicación han dejado de 'controlar' lo que se dice y el momento en el que se dice (...) Asimismo, los/las periodistas han perdido relevancia y ahora hay mucha gente que influye en la política a través de las redes sociales, al menos entre su grupo de seguidores (...) (entrevista a UPyD País Vasco)"

"Asumimos como una realidad que la información fluye, que no se controla, que se construye cada vez más desde abajo hacia arriba" (entrevista a EH Bildu)

A este respecto, la nueva estrategia de comunicación de estos partidos ha buscado también preparar al departamento para enfrentarse a la falta de rigor que provoca en ocasiones el afán de publicación inmediata o el anonimato.

\section{Fuera de campaña conviene huir de los} usos excesivamente electoralistas y explotar más las opciones de microtargeting que ofrecen los medios sociales

\subsection{Renovación de objetivos estratégicos}

En todos los partidos considerados la gestión del sitio web es interna. Según los resultados del análisis de contenido, aunque cuentan con sitios bien estructurados y con gran cantidad de información de interés, requieren prestar una mayor atención a estrategias específicas de desarrollo SEO. Es posible apreciar una ligera tendencia a priorizar los usos informativo-comunicativos, como ocurre en el caso de EAJ-PNV.eus y PPVasco.com. Aunque los usos interactivos y dialógicos ganan terrero, sólo sobresalen en el caso de EHBildu.eus/es. Por su parte, la función de movilización es predominante en el caso de Socialistasvascos.com y Upyd. es/PaísVasco (tabla 1).

Aunque los recursos destinados a proporcionar información y opinión -ya sea en formato textual, visual, audiovisual, sonoro y multimedia- han sido característicos de los websites organizacionales políticos desde sus inicios, estos evidencian un avance desde contenidos mayoritariamente textuales, a contenidos de tipo audiovisual. Las noticias y los vídeos resultan así los recursos comunicacionales más habituales en todas las webs analizadas (14,70\%), seguidos del uso de salas de prensa virtual, galerías fotográficas, reportajes, artículos y podcasts $(8,82 \%)$ (tabla 2$)$.

Como reconocen desde los departamentos de comunicación, los contenidos de actualidad encuentran un lugar destacado por su interés para el público general, así como por su utilidad para generar tráfico hacia la web, una vez son dinamizados por los usuarios en los medios sociales. Pese a su denominación, la característica sala de prensa virtual (SPV) se reconoce como un recurso o servicio a la ciudadanía, más que hacia los medios de comunicación, pues resultan contadas las ocasiones en las que los periodistas acuden a ellas en busca de una determinada información, con la excepción de contenidos concretos, como podrían ser aquellos de tipo visual y audiovisual.

La dimensión interactiva (tabla 3) se ve impulsada en buena medida por la presencia de los partidos en los medios sociales, presencia que va de la mano del propio desarrollo del

Tabla 2. Dimensión comunicación

\begin{tabular}{|l|c|r|}
\cline { 2 - 3 } \multicolumn{1}{l|}{} & $\begin{array}{r}\text { Total partidos } \\
\text { (Sí= 1; No= 0) }\end{array}$ & $\%$ \\
\hline Sala de prensa virtual & 3 & 8,82 \\
\hline $\begin{array}{l}\text { Descarga de documentos: discursos, } \\
\text { manifiestos, etc. }\end{array}$ & 2 & 5,80 \\
\hline $\begin{array}{l}\text { Comunicados/notas de prensa textuales } \\
\text { tradicionales }\end{array}$ & 1 & 2,94 \\
\hline $\begin{array}{l}\text { Comunicados/notas de prensa multime- } \\
\text { dia y 2.0 }\end{array}$ & 0 & 0,00 \\
\hline Revista-dossier de prensa & 1 & 2,94 \\
\hline Transcripción de ruedas de prensa & 0 & 0,00 \\
\hline Noticias de actualidad & 5 & 14,70 \\
\hline Reportajes y webs especiales & 3 & 8,82 \\
\hline Entrevistas & 2 & 5,80 \\
\hline Artículos de opinión & 3 & 8,82 \\
\hline Contenidos de audio/podcasts & 3 & 8,82 \\
\hline $\begin{array}{l}\text { Contenidos de vídeo (ruedas de prensa, } \\
\text { presentaciones, etc.) }\end{array}$ & 5 & 14,70 \\
\hline Canal de televisión - emisión en streaming & 2 & 5,80 \\
\hline Galerías-álbumes fotográficos & 342 \\
\hline Archivos gráficos multimedia-interactivos & 3 & 100,00 \\
\hline Total & 3 & \\
\hline
\end{tabular}


Tabla 3. Dimensión interacción

\begin{tabular}{|c|c|c|}
\hline & \\
\hline & $\begin{array}{l}\text { Total partidos } \\
(\mathrm{Sí}=1 ; \mathrm{No}=0)\end{array}$ & $\%$ \\
\hline $\begin{array}{l}\text { Suscripción a newsletters/boletines } \\
\text { electrónicos }\end{array}$ & 5 & 10 \\
\hline Alertas mail & 1 & 2 \\
\hline Registro online/perfil en el sitio & 2 & 4 \\
\hline Sindicación-RSS & 5 & 10 \\
\hline Mail con gabinete de prensa & 3 & 6 \\
\hline Mail con líderes políticos & 0 & 0 \\
\hline Votación de informaciones y noticias & 0 & 0 \\
\hline $\begin{array}{l}\text { Comentarios a informaciones de actu- } \\
\text { alidad }\end{array}$ & 2 & 4 \\
\hline (Video)chat o encuentros digitales & 1 & 2 \\
\hline Envío de sugerencias, propuestas o quejas & 2 & 4 \\
\hline $\begin{array}{l}\text { Participación en la elaboración de pro- } \\
\text { gramas electorales }\end{array}$ & 2 & 4 \\
\hline $\begin{array}{l}\text { Envío de materiales (fotos, vídeos, textos, } \\
\text { etc.) }\end{array}$ & 1 & 2 \\
\hline Participación en foros & 2 & 4 \\
\hline Participación en encuestas & 1 & 2 \\
\hline Acceso a blogs & 3 & 6 \\
\hline $\begin{array}{l}\text { Comentarios a blogs (sujetos a moder- } \\
\text { ación) }\end{array}$ & 2 & 4 \\
\hline Acceso a medios sociales & 5 & 10 \\
\hline $\begin{array}{l}\text { Opción de compartir contenidos (noticias) } \\
\text { vía web } 2.0\end{array}$ & 5 & 10 \\
\hline $\begin{array}{l}\text { Posibilidad de compartir contenidos vía } \\
\text { mail }\end{array}$ & 3 & 6 \\
\hline Visualización de contenidos sociales o 2.0 & 3 & 6 \\
\hline Alertas y mensajes vía Whatsapp & 2 & 4 \\
\hline Total & 50 & 100,00 \\
\hline
\end{tabular}

website, desde el que es posible acceder a dichos medios -mediante enlaces que aparecen en todas las páginas que componen el sitio- y visualizar contenido difundido a través de los medios sociales del partido, así como dinamizarlo. EI acceso a medios sociales y la posibilidad de compartir los contenidos vía 2.0 resultan así dos de las funciones interactivas principales en estos sitios (10\%), junto con el uso de recursos más tradicionales como el acceso a blogs de líderes, la suscripción a newsletters y boletines, o la sindicación de contenidos (RSS) (6\%). Otras opciones como los comentarios a noticias, a posts, el envío de sugerencias o la participación en la elaboración de los programas electorales resultan menos habituales (4\%), si bien todavía parece mucho más anecdótico el uso del videochat y de plataformas de upload para el envío de materiales (2\%).

La estrategia 2.0 de estos partidos comienza a estructurarse hacia el año 2007 y actualmente se encuentra en un momento de inflexión o, si se prefiere, de transición. Es más, según reconocen internamente, de entre las múltiples evoluciones que las nuevas tecnologías han impulsado en su estrategia comunicativa, el cambio de mentalidad derivado de la asimilación del 2.0 ha resultado ser hasta la fecha el más impactante. Concretamente, todos los partidos coinciden al
Tabla 4. Dimensión movilización

\begin{tabular}{|l|c|c|}
\cline { 2 - 3 } \multicolumn{1}{l|}{} & $\begin{array}{c}\text { Total partidos } \\
\text { (Sí= 1; No=0) }\end{array}$ & $\%$ \\
\hline $\begin{array}{l}\text { Formulario de afiliación para nuevos } \\
\text { miembros }\end{array}$ & 4 & 10,25 \\
\hline $\begin{array}{l}\text { Posibilidad de realizar aportaciones y } \\
\text { donativos }\end{array}$ & 1 & 2,56 \\
\hline Adquisición de merchandising & 0 & 0,00 \\
\hline $\begin{array}{l}\text { Descarga de material propagandístico: } \\
\text { programa, logotipo, himnos, carteles y } \\
\text { panfletos, etc. }\end{array}$ & 1 & 2,56 \\
\hline Historia del partido & 4 & 10,25 \\
\hline Estatutos del partido & 4 & 10,25 \\
\hline Valores e ideología-programa & 5 & 12,82 \\
\hline Biografía de líderes & 3 & 7,69 \\
\hline Estructura organizativa & 4 & 10,25 \\
\hline Resultados electorales & 1 & 2,56 \\
\hline Cuentas & 2 & 5,12 \\
\hline Calendario de eventos/agenda & 5 & 12,82 \\
\hline Información de contacto & 5 & 12,82 \\
\hline Total & & 100,00 \\
\hline
\end{tabular}

señalar las siguientes ventajas (a y b) y desventajas (c) de la comunicación 2.0 para la política:

a) inmediatez o capacidad de respuesta rápida ante determinadas situaciones, informaciones o estados de opinión;

b) humanización de los/las políticos a nivel público y también privado;

c) aumento de la sobreexposición pública, inherente per se a la labor política.

Se admite también que cada plataforma cuenta con ventajas, como la capacidad de explicación de acciones y de interactividad que otorga Facebook, o la utilidad técnica o instrumental de YouTube, como repositorio de vídeos que son compartidos también en otras redes sociales. En el uso de Google+ pesan otros criterios, como el hecho de que se trata de la red del gigante Google.

También se admiten ciertas desventajas, como el desgaste que implica Twitter, por las dificultades que acarrea esta red en muchos casos para generar un debate productivo. La estrategia en blogs resulta algo más específica, pues requiere interactividad, respuesta al usuario y actualización, algo que no siempre se consigue en todos los casos. De todos modos, los blogs son considerados una herramienta muy importante para los políticos, porque permiten profundizar en un asunto de actualidad desde una perspectiva más personal que oficialista.

Las webs analizadas evidencian también una función de movilización del electorado, observable principalmente a partir de la publicación de información relativa al programa del partido y su agenda, o la aportación de opciones para el contacto con éste $(12,82 \%)$. También destaca el uso de otro tipo de información básica, como su historia, estatutos y estructura organizativa $(10,25 \%)$ (tabla 4). 
Por lo que se refiere a la accesibilidad y navegación, destaca el uso de enlaces tanto internos como externos (12,9\%), así como de mecanismos de acceso reticular a la home del sitio (12,9\%). En menor medida, se hace uso de buscadores internos sencillos, mapas web y archivos o hemerotecas (9,67\%). Opciones como buscadores avanzados, buscadores externos o modificar el tamaño de la fuente resultan más escasas (3,22\%).

\section{Discusión y conclusiones}

En línea con la hipótesis del estudio, las organizaciones políticas del País Vasco demuestran una actitud digital y 2.0 que requiere de mayor concreción y afianzamiento, pero que evidencia ya un nuevo estilo, común a otros departamentos y organizaciones, dedicados o no a la política. El reconocimiento de la web como el nuevo espacio público les lleva a reconocer la importancia de su papel en la transmisión del mensaje político, al tiempo que muestran sus dudas sobre la capacidad efectiva de los medios sociales para ser hoy por hoy los principales generadores de opinión y dinamizadores de la vida política vasca.

El estudio ha detectado diversas evidencias del momento evolutivo a medio camino o 1.5 (Kalnes, 2009) que viven estos partidos en relación a su comunicación.

1. Los sitios web organizacionales son gestionados como espacios comunicativos autónomos dirigidos a la ciudadanía en general. Los gabinetes implicados demuestran su preferencia por los recursos útiles para generar interactividad, al mismo nivel o por encima de otros, como los de tipo informacional y de movilización política. Ello confirma que las rutinas de los departamentos examinados se han ido adaptando para interesar a los ciudadanos, más que para atraer a los periodistas. Recursos antes identificativos de las webs organizacionales como la sala de prensa virtual (SPV) han perdido impulso. Con todo, a falta de una relación 2.0 con los periodistas, tampoco la sala de prensa de medios sociales -social media newsroom (SMNR)- parece ser un recurso probable a corto plazo. De acuerdo con ello, tampoco se han introducido aún rutinas de trabajo con formatos como la nota de prensa 2.0 o las ruedas de prensa vía Twitter, por ejemplo.

2. Los partidos analizados admiten estar llevando a cabo actualmente una reflexión sobre dónde y cómo hay que estar y comunicar en los distintos espacios online para explotar la interactividad, de ahí su renovación periódica de los websites y sus constantes movimientos de unas redes sociales a otras.

\section{Se percibe cierta inquietud en relación con la validez de las plataformas online para generar opinión y diálogo}

3. Los planteamientos online han comenzado a hacerse también desde posiciones alejadas de la comunicación puramente electoral y enfocada a la obtención de votos (Norris, 2005), aunque las dificultades que dicen encontrar en la gestión del diálogo 2.0 con medios y ciudadanos haga que tiendan a usos electoralistas. De hecho, se percibe cierta inquietud en relación con la validez de las plataformas online para generar opinión y diálogo. Concretamente, se muestran preocupados por la pérdida de control, ya que los debates y las crisis son difíciles de manejar en las redes sociales, lo contrario de lo que ocurría en los medios de masas, donde el proceso de difusión e impacto del mensaje estaba mucho más controlado. 


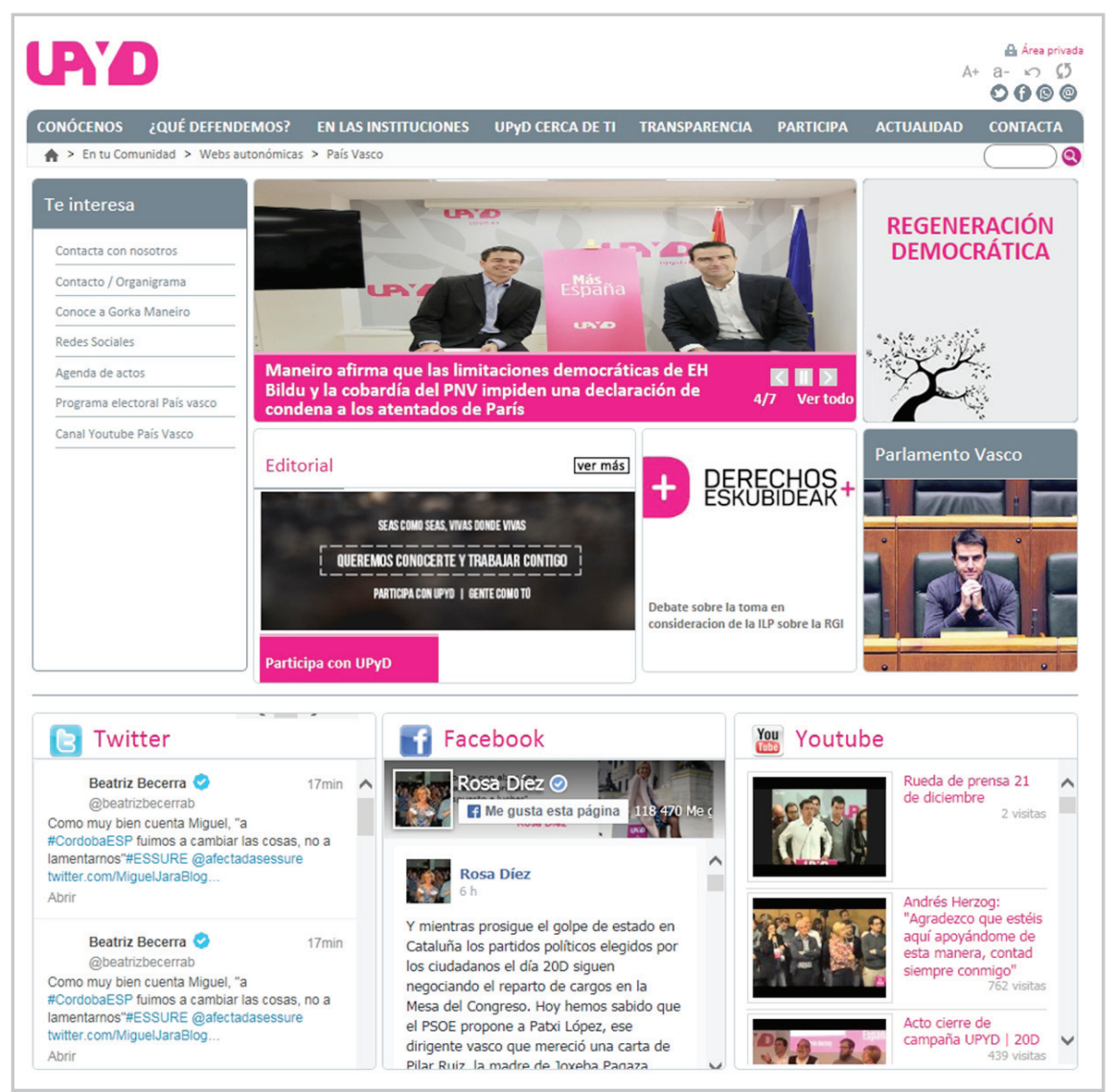

http://www.upyd.es/Pais-Vasco

4. Todos los departamentos han adaptado sus plantillas para responder a la gestión comunicativa en la web, sobre todo en lo referido a la gestión de comunidades (community management). Con todo, estos gabinetes reconocen la necesidad de perfeccionar aún más esta tarea que requiere tiempo y una alta especialización, algo que no siempre es posible. Profundizar en el diálogo con los públicos es considerado el principal reto a corto y medio plazo, aunque las propias características del medio online y el ámbito del diálogo, la política, son factores que complican esta tarea, lo que no implica que no se deban realizar mayores esfuerzos por precisar fórmulas adecuadas. Sigue pendiente también de una manera mucho más evidente la respuesta definitiva a otras necesidades como la especialización en posicionamiento estratégico (SEO y SEM) y el análisis de indicadores web. Asimismo, aunque los respectivos dircoms han aumentado su manejo de las nuevas tecnologías, ninguno admite usos excesivamente técnicos o avanzados, ni ofrece una autopercepción como dircom 2.0. Se trata en cualquier caso de una visión común, según revelan diversos estudios e informes recientes (Dircom, 2013).

Cabe deducir, en suma, que el sitio web continuará ganando terreno como herramienta informativa y como ámbito principal de comunicación de la alta política (comunicados, titulares, ruedas de prensa, etc.), mientras que las redes sociales serán el escaparate de esa actividad del partido a un nivel más cercano e informal. Esta función 2.0 de los departamentos de comunicación reforzaría su valor, especialmente en el ámbito de la política, al tratarse de un espacio emocional que suscita pasiones, aunque los políticos tiendan a pensar que la comunicación es un proceso racional en el que imperan las estadísticas. Si bien algunos partidos ya han comenzado a abrirse de manera evidente a la participación de las bases, por ejemplo en la realización de los programas electorales, resulta un hecho que es necesario experimentar más con campañas de tipo bottom-up en momentos electorales puntuales, mientras que fuera de éstos se debería huir de los usos excesivamente electoralistas y explotar más las opciones de microtargeting que ofrecen los medios sociales.

\section{Nota}

1. EH Bildu es una coalición política que integra a los partidos políticos Sortu, Eusko Alkartasuna, Aralar y Alternatiba.

\section{Agradecimientos}

Esta investigación ha sido realizada en el marco del proyecto Estrategia y gestión comunicativa 2.0 de los partidos políticos en el País Vasco. Implicaciones para medios y público (Ref. EHUA13/10, UPV/EHU), del proyecto Audiencias activas y periodismo. Análisis de la calidad y la regulación de los contenidos elaborados por los usuarios (Ref. CSO2012-39518-C04-03, Ministerio de Economía y Competitividad), y del Grupo Consolidado GIU 13/13 (UPV/EHU).

Los partidos analizados admiten estar llevando a cabo actualmente una reflexión sobre dónde y cómo hay que estar y comunicar en los distintos espacios online para explotar la interactividad

\section{Bibliografía}

Asociación de Directivos de la Comunicación (2013). Anuario de la comunicación 2013.

http://www.dircom.org/anuario2013

Blumenthal, Sidney (1980). The permanent campaign. Nueva York: Simon and Schuster. ISBN: 9780807032084

Canel, María-José; Zamora, Rocío (2004). "La comunicación en las organizaciones políticas: la estrategia permanente en el mercado de la visibilidad de los poderes públicos". En: Losada-Díaz, José-Carlos (coord.). Gestión de la comunicación en las organizaciones. Barcelona: Ariel, pp. 515-541. ISBN: 9788434413030

Codina, Lluís (2006). Metodología de análisis y evaluación de recursos digitales. 
http://www.lluiscodina.com/metodo-talleres

Dader, José-Luis; Cheng, Lifen; Campos, Eva; Quintana, Nuria; Vizcaíno-Laorga, Ricardo (2014). "Las webs de los partidos españoles en campaña electoral. Continuismo entre 2008 y 2011". Trípodos, n. 34, pp. 115-152.

http://www.tripodos.com/index.php/Facultat_Comunicacio_ Blanquerna/article/view/169

De-Landtsheer, Christ'I (2012). "Towards a methodology for evaluating the quality of political websites". En: Marczewska-Rytko, María (ed.). Democratic thought in the age of globalization. Lublin: University Maria Curie Sklodowska Press. ISBN: 9788377841419

García-Orosa, Berta (2009). Gabinetes de comunicación on line. Sevilla: Comunicación Social. ISBN: 9788496082786

Gibson, Rachel; Ward, Stephen (2000). "A proposed methodology for studying the function and effectiveness of party and candidate websites". Social science computer review, v. 18, n. 3, pp. 301-319.

http://dx.doi.org/10.1177/089443930001800306

Gibson, Rachel; Margolis, Michael; Resnick, David; Ward, Stephen (2003) "Election campaigning on the WWW in the USA and UK: A comparative analysis". Party politics, v. 9, n. 1, pp. 47-75.

http://dx.doi.org/10.1177/135406880391004

Gobierno Vasco (2012). "Percepción de la situación sociopolítica". Sociómetro vasco, n. 50, noviembre.

http://www.observatorioreligion.es/upload/16/58/ Sociometro_Vasco._Noviembre_2012._N_50.pdf

Gómez-Castellanos, Rodolfo; Ortiz-Marín, Manuel; Concepción-Montiel, Luis-Enrique (2011). "Tecnologías de la comunicación y política 2.0”. Espacios públicos, v. 14, n. 30, pp. 72-84. http://www.redalyc.org/articulo.oa? $i d=67618934006$

Gutiérrez-Rubí, Antoni (2014). Tecnopolítica. Madrid: Bebookness. ISBN: 9788469718063

http://www.gutierrez-rubi.es/tecnopolitica

Howard, Philip N. (2006). New media campaigns and the managed citizen. New York: Cambridge University Press. ISBN: 9780521612272

https://smpsebastiao.files.wordpress.com/2010/09/ebook_gcc_howard_nm-campains-and-managed-citizen.pdf

Kalnes, Øyvind (2009). "E-ruptions in party politics? The case of web 2.0 and Norwegian parties". Quaderns del CAC, n. 33, pp. 63-76.

http://www.cac.cat/pfw_files/cma/recerca/quaderns_cac/ q33_kalnes_en.pdf

Losada-Díaz, José-Carlos (2009). "Los 10 frentes de la comunicación corporativa”. Doxa comunicación, n. 6, pp. 145-164. http://dspace.ceu.es/handle/10637/5896
Macnamara, Jim; Sakinofsky, Phyllis; Beattie, Jenni (2012). "E-electoral engagement: How governments use social media to engage voters". Australian journal of political science, v. 47, n. 4, pp. 623-639.

http://dx.doi.org/10.1080/10361146.2012.731491

Norris, Pippa (2003). "Preaching to the converted?: Pluralism, participation and party websites". Party politics, v. 9, n. 1, pp. 21-45.

http://dx.doi.org/10.1177/135406880391003

Ornstein, Norman J.; Mann, Thomas E. (eds.) (2000). The permanent campaign and its future. Washington: American Enterprise Institute. ISBN: 9780844741345

Padró-Solanet, Albert; Cardenal, Ana-Sofía (2008). "Partidos y política en internet: un análisis de los websites de los partidos políticos catalanes". IDP, n. 6, pp. 46-65.

http://www.uoc.edu/idp/6/dt/esp/padro-solanet_cardenal.pdf

Schweitzer, Eva J. (2008). "Innovation or normalization in e-campaigning? A longitudinal content and structural analysis of German party websites in the 2002 and 2005 national elections". European journal of communication, v. 23, pp. 449-470.

http://dx.doi.org/10.1177/0267323108096994

Silva, Catarina (2013). "A comunicação partidária online: os websites num contexto não eleitoral". En: Serra, Paulo; Camilo, Eduardo; Conçalves, Gisela (eds.). Participação política e web 2.0. Covilha: LabCom, pp. 195-242. ISBN: 9789896541040

Spyridou, Paschalia-Lia; Veglis, Andreas (2011). "Political parties and web 2.0 tools: A shift in power or a new digital bandwagon?". International journal of electronic governance, v. 4, n. 1-2, pp. 136-155.

http://dx.doi.org'10.1504/IJEG.2011.041712

Túñez-López, Miguel (2012). La gestión de la comunicación en las organizaciones. Sevilla: Comunicación Social Ediciones y Publicaciones. ISBN: 9788492860937

Vaccari, Cristian (2008). "Italian parties' websites in the 2006 elections". European journal of communication, v. 23, n.1, pp. 69-77.

http://dx.doi.org/10.1177/0267323107085839

Valera-Ordaz, Lidia; López-García, Guillermo (2014). “Agenda y marcos en las webs de PP y PSOE en la cibercampaña de 2011". Revista latina de comunicación social, n. 69, pp. 41-66.

http://dx.doi.org/10.4185/RLCS-2014-1000

Vergeer, Maurice; Hermans, Liesbeth; Sams, Steven (2011). "Online social networks and micro-blogging in political campaingning: the exploration of a new tool and a new campaign style". Party politics, pp. 1-25.

http://dx.doi.org/10.1177/1354068811407580 


\section{Colección de libros de bolsillo}

\section{El profesional de la información (Editorial UOC)}
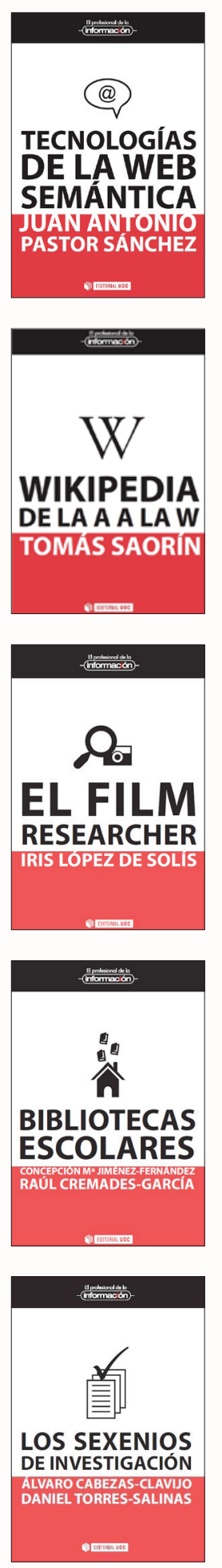
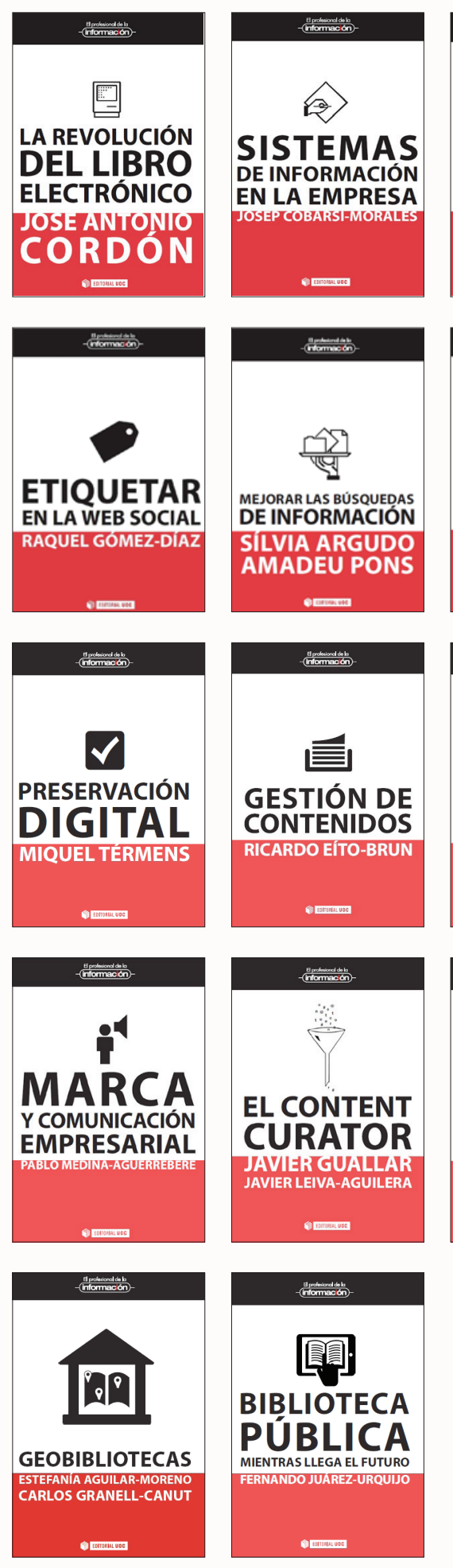

El profesional de la
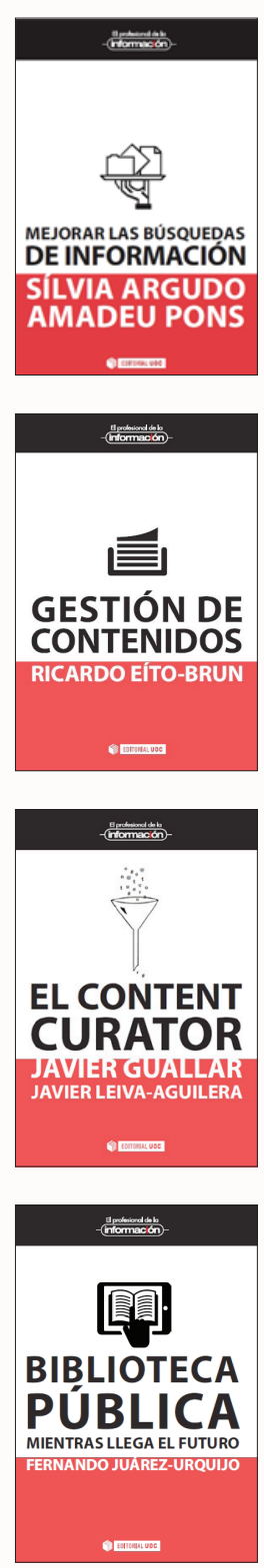
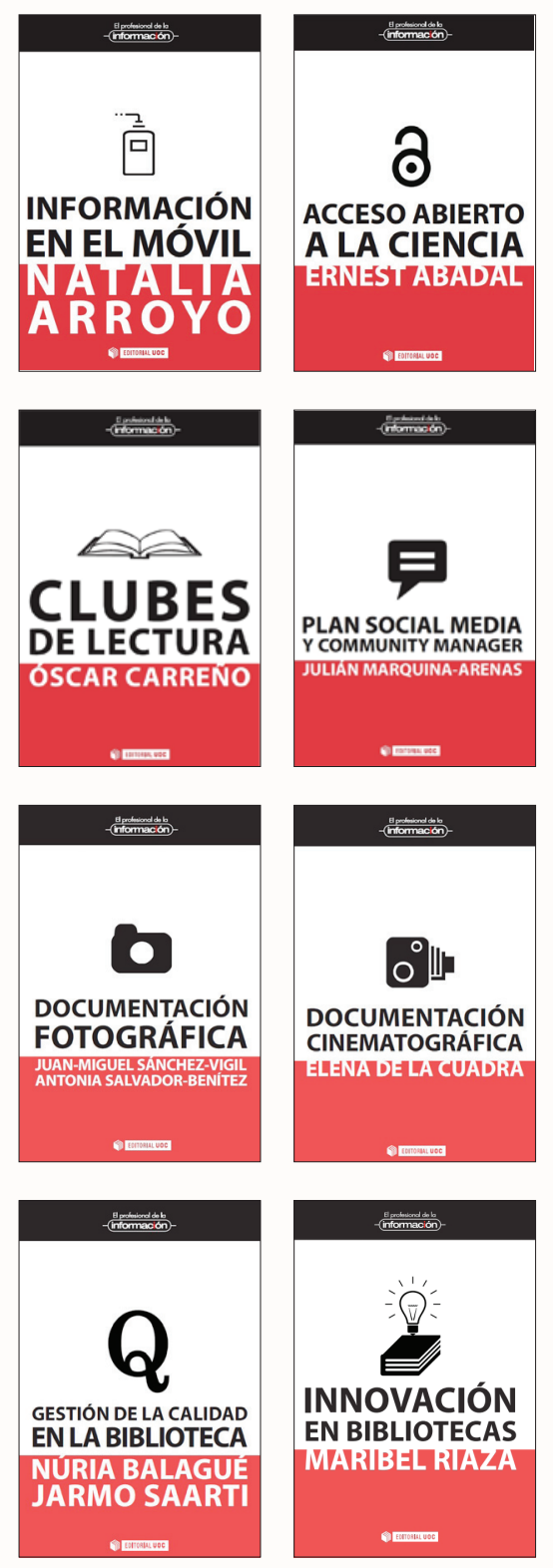
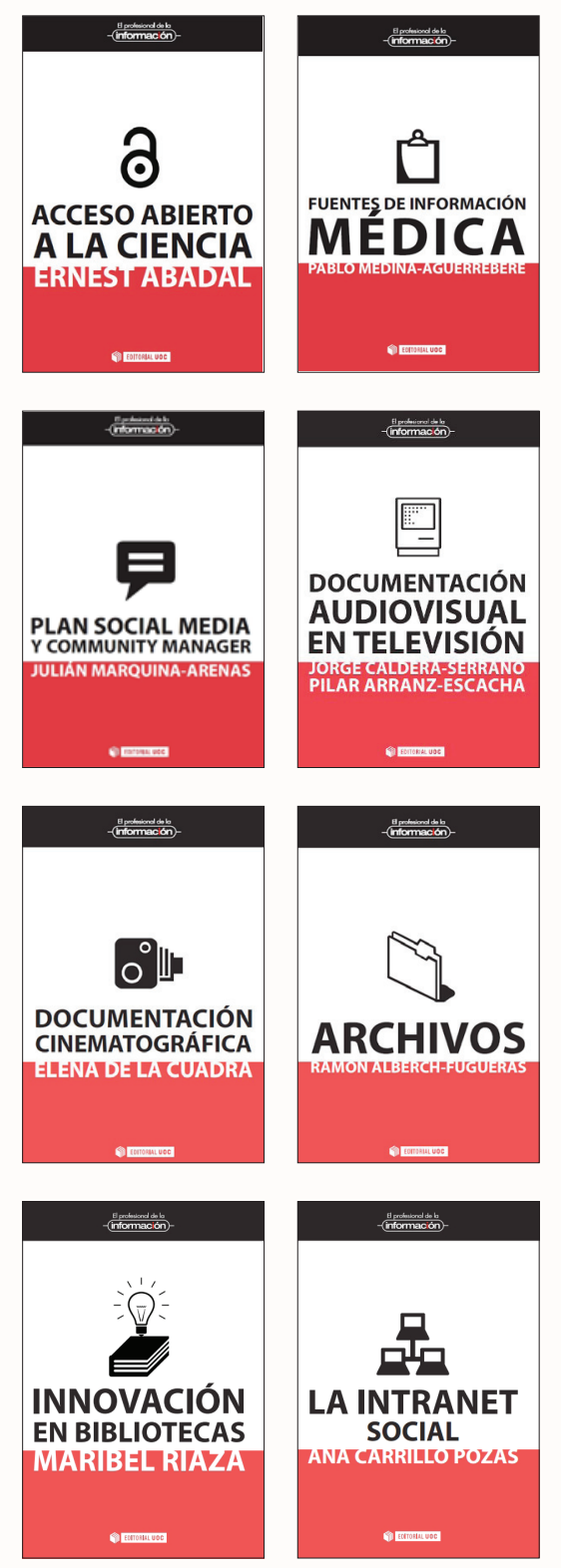
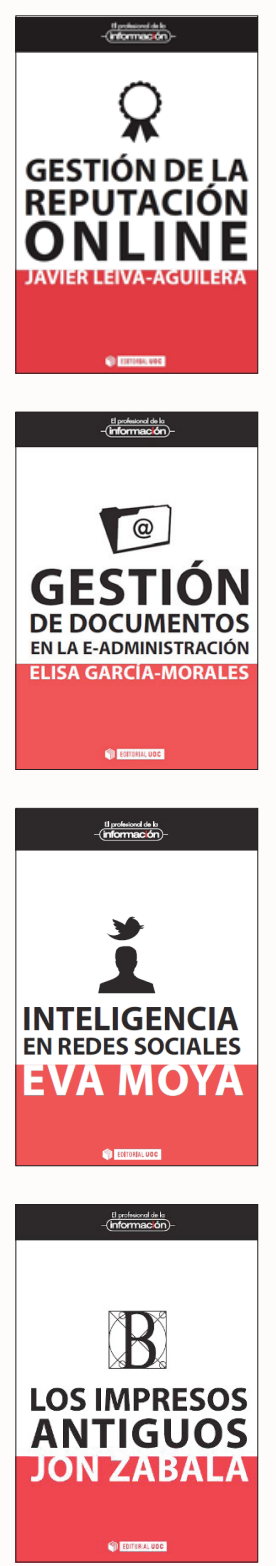

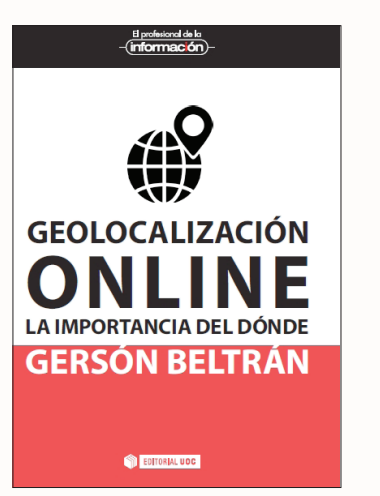

\section{Próxima}

aparición

Más información: 